\title{
ALMA EDIZIONI: UNA SFIDA NEL COMPLESSO MONDO DELLA DIDATTICA DELL'ITALIANO COME LINGUA STRANIERA
}

Ciro Massimo $\mathrm{Naddeo}^{1}$

\section{Riassunto}

In questo articolo si presenta brevemente la complessa situazione dell'insegnamento e della promozione della lingua italiana nel mondo. Successivamente viene tratteggiata la politica editoriale che Alma Edizioni nel corso degli anni ha fatto propria, in modo da sostenere e contribuire alla motivazione allo studio della lingua italiana.

Parole-chiave: Promozione, lingua italiana, motivazione allo studio dell'italiano, editoria

\section{Resumo}

O presente artigo trata brevemente da situação do ensino e da promoção da língua italiana no mundo. Sucessivamente, apresenta a política editorial que a Editora Alma, no curso dos anos, vem estabelecendo no sentido contribuir com a motivação do estudo da língua italiana, dando-lhe sustentação.

Palavras-chave: Promoção, língua italiana, motivação para o estudo de italiano, editoria

\section{CRESCITA DELLA RICHIESTA DELLA LINGUA E DELLA CULTURA ITALIANA NEL MONDO}

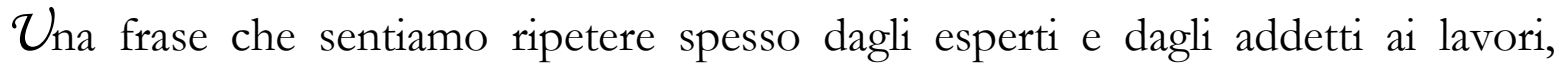
quando discutiamo della fascinazione che l'italiano ancora esercita all'estero presso studiosi, appassionati, studenti e semplici curiosi, è che la richiesta di lingua e cultura italiana nel mondo è in costante crescita.

Probabilmente, e nonostante tutto (e in questo "tutto" ci mettiamo anche la politica di promozione della lingua che di volta in volta i nostri governi attivano o, per meglio dire, non attivano), probabilmente dicevo, quest'affermazione risponde a verità. Le indagini - anche se non recentissime - ci dicono infatti che l'italiano si attesta ormai tra il quarto e quinto posto tra le lingue più studiate nel mondo.

\footnotetext{
${ }^{1}$ Direttore editoriale Alma Edizioni
} 
Ci sono naturalmente anche dei segnali contrastanti e che vanno in direzione opposta a quanto detto. È di qualche mese fa ad es. la notizia che l'unione europea esclude l'italiano dal gruppo ristretto delle lingue in cui vengono svolti i lavori e redatti i documenti ufficiali (inglese, francese e tedesco). E questo nonostante la battaglia in senso contrario che il governo italiano ha cercato di portare avanti. È evidente che ciò rischia di innescare un processo involutivo in base al quale la minor necessità d'uso (pensiamo a tutti i concorsi per addetti ai lavori della commissione che non contempleranno più l'italiano) porterà a un calo della domanda di formazione linguistica, e dunque a un possibile conseguente calo di corsi, classi, studenti.

Tra i segnali in controtendenza o che potrebbero contribuire a determinare una controtendenza si potrebbe citare poi il taglio operato dal governo in una delle ultime finanziarie ai fondi per i quotidiani e i periodici italiani all'estero, che mette in serio pericolo la sopravvivenza di storiche testate italiane prodotte in altri paesi (Germania, Stati Uniti, Venezuela, Australia, Argentina) e destinate ai nostri connazionali all'estero. Una scelta miope, vista tra l'altro l'esiguità del risparmio per le casse dello stato ( 5 milioni in tutto). Ma questo rientra in un discorso più generale sulla inadeguatezza della nostra politica di diffusione della lingua.

E comunque, dicevamo, la tendenza generale registra una crescita della richiesta di lingua e cultura italiana nel mondo, la conoscenza della nostra lingua si diffonde, l'interesse aumenta, e ciò in virtù della maggiore facilità di spostamento e di comunicazione che incrementa scambi, relazioni commerciali, economiche, culturali. Vanno poi considerati i nuovi flussi migratori verso l'Italia, che fanno registrare un aumento della presenza di stranieri sul territorio italiano e che determinano anche un aumento dell'interesse di lingua e cultura italiana in Italia (italiano come lingua seconda e non straniera).

Inevitabilmente cambiano anche le motivazioni allo studio. Se un tempo la stragrande maggioranza di chi studiava l'italiano lo faceva per interesse culturale (l'italiano come lingua di cultura), ora aumentano coloro che lo studiano per ragioni strumentali (lavoro) o di carattere più generalmente integrativo: far parte di una 
comunità di italofoni. L'italiano insomma è sempre più una lingua di comunicazione. Di conseguenza, aumentano le istituzioni - quelle ufficiali e quelle private - che si occupano di offrire formazione linguistica a tutti i livelli, e i soggetti che si occupano di promuovere la diffusione della lingua e della cultura italiana a vario titolo (oltre alle istituzioni pubbliche - istituti di cultura, comitati dante alighieri, università - anche quelle private, scuole università, ma anche associazioni, editori, ecc.).

\section{METODI E I MATERIALI}

I metodi e i materiali non risentono più di quell'arretratezza storica rispetto ai metodi e ai materiali relativi all'insegnamento delle lingue tradizionalmente più studiate, l'inglese, lo spagnolo, il francese, quelle lingue cioè che, per evidenti ragioni storiche (colonialismo) hanno una lunga tradizione di lingua seconda. Oggi possiamo dire per es. che i libri di lingua italiana per stranieri hanno lo stesso livello di qualità di quelli per le altre lingue, se non migliori in molti casi.

\section{LA SFIDA DI ALMA EDIZIONI}

Alma Edizioni è una casa editrice presente con i suoi prodotti in più di $\mathbf{8 0}$ paesi nel mondo. In moltissimi di questi paesi la casa editrice occupa una posizione di leadership nel mercato editoriale dellitaliano lingua straniera. I libri Alma sono adottati nelle principali istituzioni, università, scuole italiane e internazionali in cui si insegna italiano.

Un secondo dato: Alma Edizioni non nasce come editore puro. Il nucleo fondatore non proviene dal mondo dell'impresa ma dal mondo dell'insegnamento e della ricerca, e si è formato per anni nei corsi di lingua e cultura italiana per stranieri in Italia e all'estero, attraverso la sperimentazione nelle classi, il contatto quotidiano con gli studenti, insomma, sul campo, come si usa dire.

Riguardo al secondo aspetto, alla peculiarità di Alma come casa editrice nata dal mondo dell'insegnamento e della ricerca, ciò implica innanzitutto un'attenzione preminente al progetto culturale e pedagogico, proponendosi sostanzialmente come un vero e proprio operatore culturale. 
Dal punto di vista pedagogico ciò significa proporre materiali accomunati da una linea editoriale coerente, rigorosa dal punto di vista metodologico, e, per quanto possibile, innovativa.

Dal punto di vista culturale ciò significa assumere una prospettiva che aiuti a decifrare le tendenze della società in continua evoluzione, i cambiamenti nei costumi, negli stili di vita e nella mentalità.

Dal punto di vista didattico il concetto di motivazione, e quindi quelli di gioco, di sfida, di gusto per la scoperta, di cooperazione costituiscono le parole d'ordine cui si attengono gli autori Alma.

Il piacere insomma è la chiave che Alma ha individuato per proporre e affrontare in modo efficace argomenti seri e impegnativi come la grammatica (sintassi, morfologia, fonologia), la letteratura, la cultura.

Dai percorsi di Guastalla (2002, 2004), segnalati da taluni come una delle caratteristiche distintive della didattica dell'italiano nel panorama dell'educazione linguistica europea contemporanea (cfr. Torresan 2011: 15) alla complessità di media e dei linguaggi nel nuovissimo Domani (Guastalla, Naddeo 2010, 2011), dalla ricchezza sociolinguistica dei volumi per l'ascolto (Anzivino, D’Angelo 2009) alle ingegnose strategie per stimolare alla lettura nel volume Letture in Gioco (Mattei, Merzagora Piatti, Merzagora Piatti 2003), e così via, la produzione Alma si presenta come una ricerca di mezzi e strategie per intrigare, accattivare l'attenzione dell'apprendente, avvicinandolo allo studio della lingua senza che quasi egli si renda conto di sforzarsi, di far fatica, piuttosto coinvolgendolo sia in termini cognitivi che emotivi.

Da un punto di vista formativo, infine, Alma si propone come punto di collegamento e scambio di esperienze, mediante le riviste indirizzate al grande pubblico degli insegnanti di italiano nel mondo (Officina, disponibile sia in versione online che su carta) e di quanti operano, in particolare, nelL'ambito dell'italianistica in America Latina (Cartabianca, disponibile per ora solo su carta).

Alma edizioni è stata spesso presente a eventi indetti in terra brasiliana, mediante stand, presentazioni, contatti con gli autori. Dallo scorso gennaio 2011, ha aperto una sede a San Paolo. 
Per ulteriori informazioni contattare le dott.sse Daniela Policela (daniela@almaedizioni.it)e Beatriz Morassutti (beatriz@almaedizioni.it).

\section{Note bibliografiche}

Anzivino F., D’Angelo K. Ci vuole orecchio (3 voll.). Roma: Alma, 2009.

Guastalla C. Giocare con la letteratura. Roma: Alma, 202.

Guastalla C.Giocare con la scrittura. Roma: Alma, 2004. .

Guastalla C., Naddeo C. M. Domani 1. Roma: Alma, 209. .

Guastalla C., Naddeo C. M. Domani 2. Roma: Alma, 2009. .

Torresan P.Dove sta andando la didattica dell'italiano? Metodologia ed esperienze di frontiera". In: Mosaico Italiano, suppl. Comunità Italiana, 16, 140, 13-16, 2010. 